\title{
AN INTERESTING CASE OF HSV PNEUMONIA AND PCP CO-INFECTION IN A PATIENT WITH AIDS: A DIAGNOSTIC AND MANAGEMENT CHALLENGE
}

\author{
Mohammed Mitha ${ }^{1}, M B C h B$ \\ Kriban Reddy ${ }^{2}, M B C h B$ \\ Raveen Parboosing ${ }^{1}, M B C h B, F C P a t h$ (Virol) \\ Yacoob Coovadia ${ }^{1}$, MB ChB, FCPath (Microbiol) \\ ${ }^{1}$ Department of Medical Microbiology, University of KwaZulu-Natal, Durban, and National Health Laboratory Service \\ ${ }^{2}$ Department of Surgery, University of KwaZulu-Natal
}

The advent of HIV and AIDS has brought about many diagnostic and management challenges regarding multiple opportunistic infections. Pneumocystis jirovecii pneumonia (PCP) is a common presentation in patients with AIDS who are not on prophylaxis or highly active antiretroviral therapy (HAART). Herpes simplex 1 virus (HSV-1) is a ubiquitous virus that mainly causes benign disease during primary infection. However, it is known to cause severe pneumonia and disseminated disease in the immunocompromised. ' We present a case of HSV-1 pneumonitis and PCP co-infection in an HIV-positive patient with respiratory failure. To the best of our knowledge, based on Pubmed and Google Scholar searches, this is the first case to be reported in the English language literature.

\section{CASE PRESENTATION}

A 36-year-old HIV-positive woman with a CD4 count of 13 cells/ $\mu$ l was admitted to the intensive care unit (ICU) at King Edward VIII Hospital, Durban, with a 3-week history of shortness of breath (New York Heart Association grade IV) which had progressively worsened 4 days prior to admission. She also had a 2-day history of pleuritic chest pain and cough. There was no past history of tuberculosis (TB) or TB contact. The medical and surgical history was non-contributory. Clinically, the patient was noted to be well nourished, with a body mass index $>25$ $\mathrm{kg} / \mathrm{m}^{2}$, febrile and tachypnoeic. There were no herpetic lesions on her lips or mouth. She was in severe respiratory distress and chest auscultation revealed global crepitations with increased intensity at the bases of the lungs. The cardiac and abdominal examinations were normal. The patient was alert and orientated with no features of meningism.

Arterial blood gas analysis revealed severe type I respiratory failure with a $\mathrm{PO}_{2} 4.5 \mathrm{kpa}, \mathrm{PCO}_{2} 3.5 \mathrm{kpa}$ and oxygen saturation $75 \%$. The chest radiograph showed bilateral homogeneous opacities and a ground-glass appearance. In light of these findings the patient was intubated, ventilated and started empirically on intravenous amoxicillin-clavulanic acid and gentamycin. The haemoglobin concentration was $12.3 \mathrm{~g} / \mathrm{dl}$, the white cell count $8.0 \times 10^{9} / 1$ and the platelet count $249 \times 10^{9} / 1$. Electrolyte levels and renal function were normal. Liver function tests showed a decreased albumin level of 19 $\mathrm{g} / \mathrm{l}$ and an increased gamma-glutaryltransferase (GGT) level of $125 \mathrm{IU} / \mathrm{l}$. A bacterial and fungal septic screen was performed on blood, urine and endotracheal aspirate (ETA) samples. The diagnosis of PCP was confirmed by immunofluorescent testing (Axis; Shield Diagnostics Ltd, UK) on the ETA samples, which were also negative for routine bacterial pathogens and TB. Trimethoprim (TMP) 240 mg-sulphamethoxazole (SMX) 2400 mg 6-hourly intravenously and prednisone $40 \mathrm{mg}$ intravenously daily were started; however, over the next few days there was no improvement in her clinical condition. Blood and ETA samples were obtained to exclude infection with atypical bacteria and viruses. She was started empirically on piperacillin-tazobactam and amikacin for suspected nosocomial sepsis. Viral studies revealed a positive HSV-1 DNA polymerase chain reaction (PCR) on the ETA and two whole-blood samples. ETA samples were negative for cytomegalovirus and respiratory syncytial virus. HSV Ig M serology was negative. The patient was immediately started on acyclovir 800 mg 8-hourly intravenously approximately 1 week after the initiation of TMP-SMX and 
prednisone. TMP-SMX and prednisone were continued concurrently with acyclovir.

The patient remained on maximum ventilatory support and died 22 days after admission.

\section{DISCUSSION}

HSV-1 may cause tracheobronchitis or pneumonitis and is associated with significantly increased mortality in critically ill patients. ${ }^{2}$ It is usually due to reactivation of the virus, which occurs as a result of certain stimuli such as fever and ultraviolet light as well as immunosuppression. ${ }^{1,2}$ It is a rare cause of respiratory disease in HIV-positive individuals, the likelihood increasing with progression to AIDS; it is an even rarer cause of respiratory infection in the immunocompetent. ${ }^{3}$

There are several hypotheses regarding the pathogenesis of HSV-1 pneumonia. The virus may reach the lower respiratory tract through contiguous spread, aspiration from the oropharynx or haematogenous spread in the presence of viraemia. ${ }^{1}$ It is usually due to reactivation and not primary disease. ${ }^{2,4}$ This was consistent with the findings in our patient, in whom testing for HSV IgM $M$ was negative.

There are no pathognomonic clinical or radiological features for HSV pneumonia. A high index of clinical suspicion with appropriate laboratory testing is required for the diagnosis. Clinically, patients may present with cough, fever, dyspnoea and hypoxaemia and failure to wean off the ventilator. ${ }^{1,5}$ Chest radiographs generally demonstrate a bilateral ground-glass appearance. Pleural effusions and patchy consolidation may also be present. ${ }^{6}$ The virus may be detected by viral isolation or PCR in broncho-alveolar lavage or endotracheal aspirate specimens. However, the significance of detecting HSV in respiratory secretions remains controversial as it may represent shedding rather than active disease. ${ }^{1-3}$ Nevertheless, the presence of HSV in respiratory secretions in severely ill patients has been associated with a poor outcome and prolonged ventilation. ${ }^{3}$ The demonstration of intranuclear inclusions or Cowdry type $A$ bodies in biopsies is highly suggestive of active infection in the lung or in other tissue specimens. ${ }^{1}$ However, performing a lung biopsy in a ventilated patient is challenging and associated with complications.

Our patient could not be weaned off the ventilator, and her condition deteriorated despite appropriate antimicrobial therapy for PCP, as well as steroids which are usually beneficial in patients with severe PCP. ${ }^{7,8}$ It has been reported in the literature that the presence of HSV in blood indicates the inability of the host to limit viral replication, with dissemination occurring particularly in immunosuppressed patients. ${ }^{9}$ HSV viraemia is typically associated with interstitial pneumonia ${ }^{4,10}$ which is in keeping with that observed in our patient with two whole blood specimens being positive for HSV-1 DNA by PCR.

The paucity of literature regarding the diagnosis and management of patients who have co-infection with HSV pneumonia and PCP suggests that this condition is either rare or under-diagnosed. Considering that both HSV-1 pneumonia and PCP have similar clinical and radiological findings, we would recommend that in patients with PCP who do not respond to appropriate therapy, co-infection with HSV-1 pneumonia should be excluded. The demonstration of HSV in blood and in respiratory samples would strongly support such a diagnosis.

It is debatable whether steroid therapy exacerbated the underlying HSV pneumonia, or contributed to ventilator dependence and overall clinical deterioration in our patient. In the absence of any other data in the literature, we suggest that if co-infection is suspected an approach would be to delay steroids for 24 - 48 hours as this immunosuppressive agent may exacerbate the HSV infection. If co-infection is confirmed, acyclovir can be given before initiation of steroids. Alternatively empiric treatment with both TMP-SMX and acyclovir together with steroids can be initiated and therapy can be modified once laboratory results become available for both PCP and HSV. Laboratory results are usually available within 24 - 48 hours

The diagnosis of HSV-1 pneumonia-PCP co-infection is rare and management is currently speculative. The optimal therapy for this dual infection remains to be determined by means of greater awareness, further research and clinical trials.

\section{REFERENCES}

. Simoons-Smit AM, Kraan EM, Beishuizen A, Strack van Schijndel RJ, VandenbrouckeGrauls CM. Herpes simplex virus type 1 and respiratory disease in critically-ill patients: Real pathogen or innocent bystander? Clin Microbiol Infect 2006; 12(11): 1050-1059

2. Engelmann I, Gottlieb J, Meier A, et al. Clinical relevance of and risk factors for HSV-related tracheobronchitis or pneumonia: results of an outbreak investigation. Crit Care 2007; 11(6): R119.

3. Bruynseels $\mathrm{P}$, Jorens PG, Demey HE, et al. Herpes simplex virus in the respiratory tract of critical care patients: a prospective study. Lancet 2003; 362: 1536-1541.

4. Ramsey PG, Fife KH, Hackman RC, Meyers JD, Corey L. Herpes simplex virus pneumonia; clinical virologic and pathologic features in 20 patients. Ann Intern Med 1982: 97: 813-820

5. Eisenstein $L E$, Cunha $B A$. Herpes simplex virus pneumonia presenting as failure to wean from a ventilator. Heart Lung 2003; 32(1): 65-66

6. Aquino SL, Dunagan DP, Chiles C, Haponik EF. Herpes simplex virus 1 pneumonia: patterns on CT scans and conventional chest radiographs. J Comput Assist Tomogr 1998; 22(5): 795-800.

7. Gagnon S, Boota AM, Fischl MA, Baier H, Kirksey OW, La Voie L. Corticosteroids as adjunctive therapy for severe Pneumocystis carinii pneumonia in the acquired immunodeficiency syndrome. A double-blind, placebo-controlled trial. N EnglJ Med 1990: 323: 1444-1450.

8. Bozzette SA, Sattler FR, Chiu J, Wu AW, Gluckstein, D, Kemper C, et al. A controlled trial of early adjunctive treatment with corticosteroids for Pneumocystis carinii pneumonia in the acquired immunodeficiency syndrome. California Collaborative Treatment Group. N Engl J Med 1990; 323: 1451-1457.

9. Whitley RJ. Herpes simplex viruses. In: Knipe DM, Howley PM, eds. Fields Virology, Vol. 2. 4th ed. Philadelphia: Lippincott Williams \& Wilkins, 2001: 2467-2468.

10. Groeneveld AB, Vandenbroucke-Grauls CM. One swallow does not make a summer: can herpes simplex virus-1 cause pneumonia and acute lung injury? Am J Respir Crit Care Med 2007; 175(9): 865-866. 